Pacific Journal of Mathematics

A PROBLEM ON CONTINUOUS AND PERIODIC FUNCTIONS 


\section{A PROBLEM ON CONTINUOUS AND PERIODIC FUNCTIONS}

\section{J. S. HwANG}

Let $f(x)$ be continuous and of period one on the real line. If $d_{j}$, $j=1,2, \ldots, n$, are $n$ numbers such that each $d_{j}-d_{1}$ is rational, then there are two rational numbers $r$ and $r^{\prime}$ for which

$$
f(r) \leq f\left(r+d_{j}\right) \text { and } f\left(r^{\prime}\right) \geq f\left(r^{\prime}+d_{j}\right), \quad j=1,2, \ldots, n .
$$

This problem was communicated to the author by $K$. L. Chung and P. Erdös.

1. Introduction. Let $f(x)$ be a real valued function. We say that $f(x)$ is of period one if

$$
f(x+1)=f(x) \text { for }-\infty<x<\infty .
$$

A problem (communicated by Chung and Erdös) asks that if $f(x)$ is continuous and of period one, and if $d_{j}, j=1,2, \ldots, n$, are $n$ numbers, can one find a rational number $r$ such that

$$
f(r) \leq f\left(r+d_{j}\right), \quad j=1,2, \ldots, n .
$$

In this note, we present the following partial solution.

THEOREM 1. Let $f(x)$ be continuous and of period one. If $d_{j}, j=$ $1,2, \ldots, n$, are $n$ numbers such that each $d_{j}-d_{1}$ is rational, then there are two rational numbers $r$ and $r^{\prime}$ for which

(1) $f(r) \leq f\left(r+d_{j}\right)$ and $f\left(r^{\prime}\right) \geq f\left(r^{\prime}+d_{j}\right), \quad j=1,2, \ldots, n$.

2. Uniform distribution. Let $x$ be a positive number and let $[x]$ be the largest integer less or equal to $x$. By a theorem of Hardy and Wright [1, Theorem 445], we know that if $\theta$ is irrational then the points $(n \theta)=n \theta$ $-[n \theta]$ are uniformly distributed in $(0,1)$. In particular, the points $(n \theta)$ are dense in $(0,1)$. Based on this theorem, we shall prove the following result. 
LEMMA 1. Let $d_{1}$ be irrational and let $d_{j}-d_{1}$ be rational, $j=2,3, \ldots, n$. If $I_{j}(k), j=1,2, \ldots, n$, are non-negative integral valued functions such that

$$
\sum_{j=1}^{n} I_{j}(k)=k, \text { for each } k=1,2, \ldots,
$$

then the points $\left(a_{k}\right)$ are dense in $(0,1)$, where

$$
a_{k}=\sum_{j=1}^{n} I_{j}(k) d_{j}, \quad \text { for } k=1,2, \ldots
$$

Proof. According to the first hypothesis, we may write

$$
d_{j}=d_{1}+p_{j} / q_{j} \text {, for some integers } p_{j} \text { and } q_{j} \text {. }
$$

This together with the second hypothesis (2) yields that

$$
\sum_{j=1}^{n} I_{j}(k) d_{j}=k d_{1}+\sum_{j=1}^{n} I_{j}(k) p_{j} / q_{j} .
$$

Multiplying the product $\Pi_{1}^{n} q_{j}$ on both sides, we obtain the assertion from Hardy and Wright's theorem.

3. Proof of Theorem 1. Let $f(x)$ be continuous and of period one. We shall prove the first set of inequalities in (1). For this, we let $m$ be the minimum of $f(x)$ and let $S_{m}$ be the set of all minimum points in $(0,1)$, i.e.

$$
S_{m}=\{x: f(x)=m, 0<x<1\} .
$$

We then have two cases to be considered: either there is a point $y \in S_{m}$ such that

$$
f(y)<f\left(y+d_{j}\right), \quad \text { for all } j=1,2, \ldots, n ;
$$

or for each $x \in S_{m}$, there is a $j=j(x)$ such that

$$
f(x)=f\left(x+d_{j}\right), \quad 1 \leq j \leq n .
$$

If the first case occurs, then there is a $\delta>0$ such that (3) holds for each $z$ in $|z-y|<\delta$. By choosing a rational number $r$ in $|r-y|<\delta$ we obtain the desired result. Therefore only the second case needs to be settled in the sequel. In this case, by applying (4) successively, we obtain a sequence of points in $S_{m}$ as follows:

$$
x, x+d_{j_{1}}, x+d_{j_{1}}+d_{j_{2}}, \ldots
$$

Clearly, this sequence (5) can be represented by

$$
x_{k}=x+\sum_{j=1}^{n} I_{j}(k) d_{j}=x+a_{k}, \quad k=1,2, \ldots,
$$

where $I_{j}(k)$ and $a_{k}$ are defined in Lemma 1. If $d_{1}$ is irrational, then by Lemma 1 the points $\left(x_{k}\right)$ are dense in $(0,1)$. It follows from the continuity 
of $f(x)$ that the function $f(x)=m$ for each $x \in(0,1)$. This clearly yields the assertion.

It remains to consider the case that $d_{1}$ is rational. Let $x_{k}$ be a number of the form defined in (6) and let $S_{x}$ be the set of all points $\left(x_{k}\right)$ in $(0,1)$, $k=1,2, \ldots$ Clearly, this set $S_{x}$ is a subset of $S_{m}$. As before, we write

$$
d_{j}=p_{j} / q_{j}, \quad j=1,2, \ldots, n, \quad \text { and } \quad Q=\prod_{1}^{n} q_{j} .
$$

Then the set $S_{x}$ contains at most $Q$ points.

We now begin with the first minimum

$$
f\left(d_{j_{1}}\right)=\min _{1 \leq j \leq n} f\left(d_{J}\right), \quad \text { for some } 1 \leq j_{1} \leq n .
$$

If this minimum point $r=d_{j_{1}}$ satisfies (1) we are done, otherwise, we consider the second minimum

$$
f\left(d_{j_{1}}+d_{j_{2}}\right)=\min _{1 \leq j \leq n} f\left(d_{j_{1}}+d_{j}\right)<f\left(d_{j_{1}}\right) .
$$

Again, if $r=d_{J_{1}}+d_{j_{2}}$ satisfies (1), we are done, otherwise, we consider the third minimum

$$
f\left(d_{j_{1}}+d_{j_{2}}+d_{J_{3}}\right)=\min _{1 \leq j \leq n} f\left(d_{j_{1}}+d_{j_{2}}+d_{J}\right)<f\left(d_{j_{1}}+d_{J_{2}}\right)<f\left(d_{J_{1}}\right) .
$$

Since the set $S_{x}$ contains at most $Q$ points, there are two positive integers $M<N \leq Q$ such that

$$
f\left(d_{j_{1}}+\cdots+d_{j_{M}}\right)=f\left(d_{j_{1}}+\cdots+d_{j_{N}}\right)<\cdots<f\left(d_{j_{1}}+\cdots+d_{j_{M}}\right) \text {, }
$$

which is absurd. This proves the first set of inequalities in (1).

Similarly, by replacing minimum by maximum, we obtain the second set of inequalities in (1). This completes the proof.

4. Finite set of extreme points. In view of Theorem 1, we may ask the question as to whether the condition that each $d_{j}-d_{1}$ be rational can be replaced by some suitable conditions on the set of minimum or maximum points. For this, we prove the following

THEOREM 2. Let $f(x)$ be continuous and of period one, and let $d_{j}$, $j=1,2, \ldots, n$ be $n$ numbers. If the set of minimum and maximum points of $f(x)$ in $(0,1)$ is finite, then there are two rational numbers $r$ and $r^{\prime}$ satisfying $(1)$.

Proof. As before, we let $m$ be the minimum of $f(x)$ and let $S_{m}$ be the set of all minimum points in $(0,1)$. Then by the hypothesis the cardinality $\left|S_{m}\right|$ of $S_{m}$ is finite. As before, we have two cases described in (3) and (4). The first case gives the assertion immediately while the second case 
implies a sequence of points in $S_{m}$ defined in (5). It follows that there are two positive integers $M<N \leq\left|S_{m}\right|$ such that

$$
f\left(x+d_{j_{1}}+\cdots+d_{j_{M}}\right)=f\left(x+d_{j_{1}}+\cdots+d_{j_{N}}\right) .
$$

We now let $\delta$ be the minimum distance between any two points in $S_{m}$. We then consider a point $y \in S_{m}$ and a rational number $r_{1}$ with $y<r_{1}<y$ $+\delta$. If this number $r_{1}$ satisfies (1), we are done, otherwise, we have

$$
f\left(r_{1}\right)>f\left(r_{1}+d_{j_{1}}\right), \quad \text { for some } 1 \leq j_{1} \leq n .
$$

By the continuity of $f(x)$, there is a $0<\delta_{1} \leq \delta / 2$ such that

$$
f(x)>f\left(x+d_{j_{1}}\right), \text { for each } x \text { in }\left|x-r_{1}\right|<\delta_{1} .
$$

Choose a rational number $r_{2}$ with $\left|r_{2}-r_{1}-d_{j_{1}}\right|<\delta_{1} / 2$. If $r_{2}$ satisfies (1), we are done again, otherwise, we have

$$
f\left(r_{2}\right)>f\left(r+d_{j_{2}}\right), \text { for some } 1 \leq j_{2} \leq n .
$$

Again, there is a $0<\delta_{2}<\delta_{1} / 2$ such that

$$
f(z)>f\left(z+d_{j_{2}}\right), \quad \text { for each } z \text { in }\left|z-r_{2}\right|<\delta_{2} .
$$

We now write

$$
z=\left(z-d_{j_{1}}\right)+d_{j_{1}}=x+d_{j_{1}}
$$

Then we have

$$
\left|x-r_{1}\right|=\left|z-r_{2}+r_{2}-r_{1}-d_{j_{1}}\right|<\delta_{1} .
$$

It follows from (8) and (9) that

$$
f(x)>f\left(x+d_{j_{1}}\right)>f\left(x+d_{j_{1}}+d_{j_{2}}\right) .
$$

Continuing this process, we finally obtain

$$
\begin{aligned}
f(x) & >f\left(x+d_{j_{1}}\right)>\cdots>f\left(x+d_{j_{1}}+\cdots+d_{j_{M}}\right) \\
& >\cdots>f\left(x+d_{j_{1}}+\cdots+d_{j_{M}}+\cdots+d_{j_{N}}\right),
\end{aligned}
$$

which contradicts (7). This concludes the existence of a rational number $r$ satisfying the first set of inequalities in (1). By the same argument, we obtain another rational number $r^{\prime}$ satisfying the second set of inequalities in (1). This completes the proof.

5. Problem. In closing this note, let us pose the following

Problem. If $d_{1}$ and $d_{2}$ are two independent numbers, and if $I_{1}(k)$ and $I_{2}(k)$ are non-decreasing, is it true that the points

$$
\left(a_{k}\right) \text {, where } a_{k}=I_{1}(k) d_{1}+I_{2}(k) d_{2},
$$

are dense in a subinterval of $(0,1)$. 
Note that the monotonicity of $I_{1}(k)$ and $I_{2}(k)$ in the above problem is simply meant that we take either $d_{1}$ or $d_{2}$ after each term in the sequence $a_{k}$. However, if this hypothesis is omitted then there does exist a sequence $\left(a_{k}\right)$ which tends to any prescribed number $0 \leq \delta \leq 1$. The following construction is due to Komlos:

For each positive integer $k$, we choose a positive integer $i_{k} \leq k$ such that

$$
\varepsilon_{k}=\left|\left(i_{k} d+k d_{2}-\delta\right)\right|=\min _{1 \leq i \leq k}\left|\left(i d+k d_{2}-\delta\right)\right|
$$

where $d=d_{1}-d_{2}$. Since $d_{1}$ and $d_{2}$ are independent, the difference $d$ is an irrational number and hence the sequence $(i d)$ forms an $\varepsilon$-net in the sense that for any $x$ there is a positive integer $i$ for which $|(i d-x)|<\varepsilon$. In particular, when $x=\delta-k d_{2}$ there is an integer $I(\varepsilon)$ such that for each $k \geq I(\varepsilon)$ there exists an $i_{k} \leq k$ satisfying $\left|\left(i_{k} d-x\right)\right|<\varepsilon$. This in turn implies that $\varepsilon_{k}<\varepsilon$ and therefore $\varepsilon_{k} \rightarrow 0$ as $k \rightarrow \infty$. We now let $I_{1}(k)=i_{k}$ and $I_{2}(k)=k-i_{k}$. Then we obtain

$$
a_{k}=I_{1}(k) d_{1}+I_{2}(k) d_{2}=i_{k} d+k d_{2}=\delta \pm \varepsilon_{k} \rightarrow \delta,
$$

which is the desired result.

Also note that this example shows that the condition that $d_{j}-d_{1}$ be rational is essential in Lemma 1.

\section{REFERENCES}

[1] G. H. Hardy and E. M. Wright, The Theory of Numbers, Oxford Univ. Press, 1945.

Received March 18, 1983. The author is indebted to Chung, Erdös, and Komlos for their many valuable comments in this research.

INSTITUTE OF MATHEMATICS

ACADEMIa Sinica

TAIPEI, TAIWAN

Current Address: Michigan State University

East Lansing, MI 48824 



\section{PACIFIC JOURNAL OF MATHEMATICS EDITORS}

DONALD BABBITT (Managing Editor)
University of California
Los Angeles, CA 90024
CHARLES R. DePrima
California Institute of Technology
Pasadena, CA 91125
R. FinN
Stanford University
Stanford, CA 94305

HERMANN FLASCHKA

University of Arizona

Tucson, AZ 85721

RAMESH A. GANGOLLI

University of Washington

Seattle, WA 98195

ROBION KIRBY

University of California

Berkeley, CA 94720

C. C. MOore

University of California

Berkeley, CA 94720
Hugo RossI

University of Utah

Salt Lake City, UT 84112

H. SAMELSON

Stanford University

Stanford, CA 94305

HaRold STARK

University of California, San Diego

La Jolla, CA 92093

\section{ASSOCIATE EDITORS}
R. ARENS
E. F. BECKENBACH (1906-1982)
B. H. NEUMANN
F. WOLF
K. YoshidA

\section{SUPPORTING INSTITUTIONS}

UNIVERSITY OF ARIZONA

UNIVERSITY OF BRITISH COLUMBIA

CALIFORNIA INSTITUTE OF TECHNOLOGY

UNIVERSITY OF CALIFORNIA

MONTANA STATE UNIVERSITY

UNIVERSITY OF NEVADA, RENO

NEW MEXICO STATE UNIVERSITY

OREGON STATE UNIVERSITY
UNIVERSITY OF OREGON

UNIVERSITY OF SOUTHERN CALIFORNIA

STANFORD UNIVERSITY

UNIVERSITY OF HAWAII

UNIVERSITY OF TOKYO

UNIVERSITY OF UTAH

WASHINGTON STATE UNIVERSITY

UNIVERSITY OF WASHINGTON 


\section{Pacific Journal of Mathematics}

\section{Vol. 117, No. $1 \quad$ January, 1985}

Amos Altshuler and Leon Steinberg, The complete enumeration of the

4-polytopes and 3 -spheres with eight vertices $\ldots \ldots \ldots \ldots \ldots \ldots \ldots \ldots$

Michael James Beeson, The $6 \pi$ theorem about minimal surfaces . . . . . . . . 17

Jeffrey Lawrence Caruso and Stefan Waner, An approximation theorem

for equivariant loop spaces in the compact Lie case ...............27

Jo-Ann Deborah Cohen, Topologies on the quotient field of a Dedekind

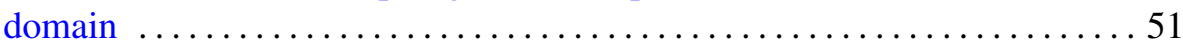

Szymon Dolecki, Gabriele H. Greco and Alois Andreas Lechicki,

Compactoid and compact filters .........................6 69

Roger William Hansell (Sr.), Generalized quotient maps that are

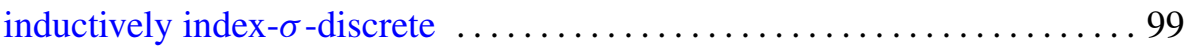

Gerhard Huisken, Capillary surfaces over obstacles $\ldots \ldots \ldots \ldots \ldots \ldots \ldots 121$

Jun Shung Hwang, A problem on continuous and periodic functions . . . . 143

Ronald Fred Levy and Michael David Rice, The extension of equi-uniformly continuous families of mappings $\ldots \ldots \ldots \ldots \ldots \ldots 149$

Kevin Mor McCrimmon, Derivations and Cayley derivations of generalized

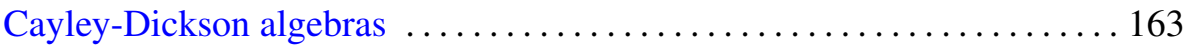

H. M. (Hari Mohan) Srivastava, A multilinear generating function for the Konhauser sets of biorthogonal polynomials suggested by the Laguerre

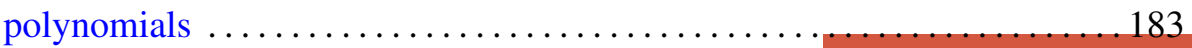

Zhu Jia Lu, Some maximum properties for a family of singular hyperbolic operators 